\title{
Initial Measurements On Pixel Detector Modules For The ATLAS Upgrades
}

\author{
Christian Gallrapp \\ CERN, Genève, Switzerland \\ on behalf of the ATLAS IBL, PPS and 3D collaborations
}

\begin{abstract}
Sophisticated conditions in terms of peak and integrated luminosity in the Large Hadron Collider (LHC) will raise the ATLAS Pixel Detector to its performance limits. Silicon planar, silicon 3D and diamond pixel sensors are three possible sensor technologies which could be implemented in the upcoming Pixel Detector upgrades of the ATLAS experiment. Measurements of the IV-behavior and measurements with radioactive Americium-241 and Strontium-90 are used to characterize the sensor properties and to understand the interaction between the ATLAS FE-I4 front-end chip and the sensor. Comparisons of results from before and after irradiation, which give a first impression on the charge collection properties of the different sensor technologies are presented.
\end{abstract}

Index Terms-ATLAS upgrade, Diamond sensors, Pixel Detector, Radiation hardness, Silicon sensors

\section{INTRODUCTION}

$\mathbf{T}$ HE need for higher luminosity and higher energies in High Energy Physics pushes the development of new technologies and systems at the LHC and the four incorporated experiments at CERN. In order to cope with these increased demands an upgrade program composed of three upgrade phases was developed which will ensure the accomplishment of the foreseen physics program. These phases can be distinguished by upgrades for maximum performance without hardware changes, upgrades with hardware changes which do not affect LHC and upgrades with major hardware changes to the whole system [1].

One of the hardware upgrades in the ATLAS experiment concerns the Inner Detector in particular the Pixel Detector and will take place during the 2013 shutdown of the LHC. The ATLAS Pixel Detector is built by three layers of pixel sensors which cover the beam-pipe in a radius of $50.5 \mathrm{~mm}$, $88.5 \mathrm{~mm}$ and $122.5 \mathrm{~mm}$. Simulations based on the expected integrated luminosity of $300 \mathrm{fb}^{-1}$ at the end of lifetime for the existing Pixel Detector show that the innermost pixel layer - the so called B-layer - will be exposed to a total ionizing dose (TID) of $47 \mathrm{Mrad}$ and a non-ionizing energy loss (NIEL) of $8 \cdot 10^{14} \mathrm{n}_{\mathrm{eq}} \mathrm{cm}^{-2}$ [2]. Given that the applied front-end chip (FE-I3) and planar pixel sensor in the ATLAS experiment were tested up to a TID of $50 \mathrm{Mrad}$ and a NIEL of $1 \cdot 10^{15} \mathrm{n}_{\mathrm{eq}} \mathrm{cm}^{-2}$ the Pixel Detector might show radiation induced degradation effects when the experiment exceeds the $300 \mathrm{fb}^{-1}$.

Knowing that a potential loss of pixel channels will effect the tracking and b-tagging capabilities of the Pixel Detector it was decided to introduce an additional pixel-layer in the existing
Pixel Detector. This upgrade stage, the Insertable B-Layer (IBL) intends to implement a new layer at a smaller radius (about $32 \mathrm{~mm}$ ) from the interaction point which will mitigate the influence of the radiation effects. The smaller radius of the Insertable B-Layer (IBL) increases the tracking capabilities of the Pixel Detector with a fourth space point and enhances the tracking performance of the whole ATLAS Inner Detector.

Among other things the upgrade efforts for the IBL detector modules concern the new front-end electronic chip (FE-I4) and three new sensor technologies which are capable of working in an environment with a peak luminosity of $2.2 \cdot 10^{34} \mathrm{~cm}^{-2} \mathrm{~s}^{-1}$. The short proximity to the interaction point increases the requirements for the front-end chip and the deployed sensor technology up to a TID of $250 \mathrm{Mrad}$ and a NIEL of $5 \cdot 10^{15} \mathrm{n}_{\mathrm{eq}} \mathrm{cm}^{-2}[2]$.

\section{FRONT-END CHIP}

The present front-end chip in the ATLAS Pixel Detector, the FE-I3 chip, is based on a $0.25 \mu \mathrm{m}$ IBM CMOS6SF technology and consists of $18 \cdot 160=2880$ readout channels corresponding to pixels with a typical size of $50 \cdot 400 \mu \mathrm{m}^{2}$ [3]. It was required for the operation in the ATLAS experiment that the chip withstands a TID of $50 \mathrm{Mrad}$ which was tested prior to the commissioning of the Pixel Detector. Three major shortcomings lead to the rejection of the FE-I3 chip as read-out chip for the IBL project. Simulations have shown that the FE-I3 chip is not able to cope with the increasing hit multiplicity expected for higher beam intensities and a shorter distance to the interaction point in the experiment [4]. The ratio between the whole FE-I3 module area and active module area is not good enough to fulfill the geometrical requirements for a layer as the IBL [2]. The requirements concerning the TID for the IBL are set to 250 Mrad which is five times higher than the design radiation dose for the FE-I3 chip.

The new developed front-end chip (FE-I4) consists of $80 \cdot 336=26880$ readout channels with a reduced pixel size of $50 \cdot 250 \mu \mathrm{m}^{2}$ compared to the FE-I3 chip. The vendor IBM is using a $130 \mathrm{~nm}$ bulk CMOS process to build the FE-I4 chips. The chip uses an amplification stage in each pixel consisting of two amplifiers to increase the gain of the amplitude and to adjust the shape of the measured charge signal. A discriminator with an adjustable threshold at the end 
of the pixel readout converts this charge signal in a time over threshold (ToT) signal with a 4-bit resolution. The firing time and the ToT value are stored with a $25 \mathrm{~ns}$ time resolution which is equivalent to one ToT unit and one bunch crossing in the LHC.

One challenge for the FE-I4 chip is to resist a higher TID up to $250 \mathrm{Mrad}$. This requirement is dealt with by using the $130 \mathrm{~nm}$ production process which provides an intrinsic radiation hardness. Due to that a radiation hardness of more than the expected $250 \mathrm{Mrad}$ can be realized in the FE-I4 frontend chip.

\section{Sensor Technologies}

The present pixel sensor technology is a $250 \mu \mathrm{m}$ thick planar silicon sensor with a $n$-in-n design. A minimum ionizing particle (MIP) passing through an unirradiated sensors creates a most probable charge of about $19 \mathrm{ke}^{-}$. The performance of the ATLAS Pixel Detector during the first years of operation have shown that it is possible to reach resolutions in the order of $10 \mu \mathrm{m}$ [3].

Due to the little space between the new introduced beam-pipe and the existing B-Layer it is not possible to shingle the IBL modules in direction of the beam as it was done for the present modules. In order to compensate for this restriction, future sensor technologies started working on different sensor layouts with the aim to decrease the inactive area at the borders of the sensors.

Three sensor technologies have shown that they are candidates to fulfill the requirements for the utilization as Pixel Detectors in the ATLAS upgrade and IBL: silicon planar pixel sensors (PPS), silicon 3D pixel sensors and diamond pixel sensors.

\section{A. Planar Pixel Sensors}

Due to the experience with the ATLAS Pixel Detector ATLAS Planar Pixel Sensors (PPS) are a well established technology. Therefore, the PPS design for IBL is based on the layout of the sensors which are currently used in the ATLAS experiment.

Two planar n-in-n design options are available as IBL upgrade candidates. The conservative design uses a pixel and guard ring layout similar to the one in the present Pixel Detector. The slim edge design uses an enhanced edge layout compared to the conservative design.

Several studies and simulations have been performed to decrease the inactive area of the planar pixel modules. 16 guard rings with a width of about $600 \mu \mathrm{m}$ are used in the present pixel modules to manage the potential drop between the cutting edge of the sample and the electrode. Additional $500 \mu \mathrm{m}$ are spent as a safety margin around the guard ring region in order to protect the guard ring structure from possible damages due to the dicing [3] [5]. Based on simulations to reduce the inactive area of the sensor a reduced number of 13 guard rings with a safety margin of $100 \mu \mathrm{m}$ was proposed for both IBL sensor designs [6] [7].

The slim edge design shown in Figure 1 uses $500 \mu \mathrm{m}$ long edge pixel which overlap eleven guard rings thus decreasing the inactive area by $15 \%$ down to $10 \%$ compared to the present pixel modules [3] [2]. Within this overlap an underdepletion zone is formed which is still wide enough depleted to measure sufficient charge to be considered as active area. Due to the shorter pixel length of $250 \mu \mathrm{m}$ in the conservative design the whole guard ring region has to be considered as inactive area.

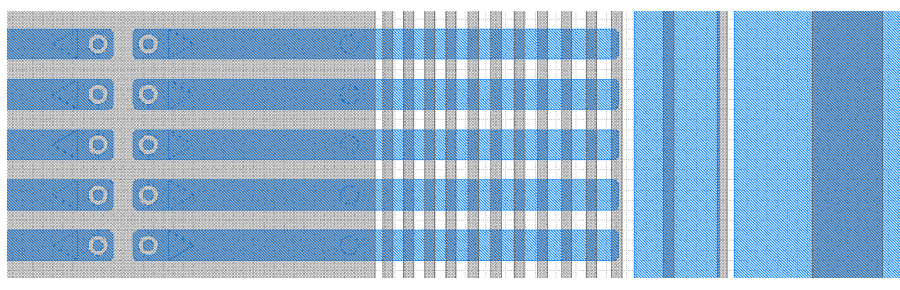

Fig. 1. Edge pixel layout of a slim edge planar pixel design, showing the 13 guard rings with the overlapping pixel region [8]

Another potential upgrade candidate is the n-in-p planar pixel design which combines different advantages but also brings new challenges. The increased radiation hardness of $n$-in-p silicon devices is a characteristic of these devices which qualifies this technology for the operation in highly irradiated environments. Additionally no type inversion appears for irradiated n-in-p devices. As the layout of these sensors applies only a single sided production process with less steps as for the n-in-n design this might bring a financial benefit for this technology. In order to gain from this feature the guard rings have to be placed on the pixellated side of the sensor. Hence the full bias voltage is right at the edge of the sensor and only a couple of $\mu \mathrm{m}$ away from the front-end electronic chip. Due to this short distance and to the high bias voltages used for planar sensors sparks might be created which would destroy the chip. N-in-p sensors produced by $\mathrm{CiS}{ }^{1}$ (Erfurt, Germany) use an additional passivation layer of benzocyclobutene to prevent the occurrence of this effect [9].

The biggest challenge for all planar pixel designs is the stable operation of the samples. This includes the operation at a stable bias current for a given temperature. In terms of the IBL requirements $5 \cdot 10^{15} \mathrm{n}_{\mathrm{eq}} \mathrm{cm}^{-2}$ irradiated planar pixel samples have to withstand the biasing of $-1000 \mathrm{~V}$ at a sensor temperature of $-15^{\circ} \mathrm{C}$.

For the planar pixel sensor IBL design the PPS collaboration proposed a $200 \mu \mathrm{m}$ thick $n-i n-n$ slim edge design. This design provides a better radiation hardness due to the higher electric field and a bigger active area due to the slim edge design.

\section{B. 3D Pixel Sensors}

3D sensors were introduced as a new architecture for solid-state radiation detectors with an intrinsic radiation hardness which is related to the shorter electrode distance than in traditional silicon detectors [10]. The advantage of this sensor technology is the independence of the sensor

\footnotetext{
${ }^{1} \mathrm{CiS}:$ http://www.cismst.org/en/
} 
thickness and the electrode distance. This property of 3D sensors makes it possible to adjust the strength of the electric field and with that also the charge collection by changing the distance of the electrodes.

The ATLAS 3D collaboration works on a single sided full 3D design with full through electrodes and a double sided 3D design with partially or full through electrodes for the IBL upgrade. Both designs are based on a n-in-p technology which was analyzed for different electrode configurations. Since the electrode configuration directly changes the electrode distance, the applied configuration has to weight the advantages and disadvantages concerning the charge collection as well as the introduced pixel noise due to capacitance. For the IBL project a '2E-250' electrode configuration has been considered as the most suitable configuration. This configuration covers $250 \mu \mathrm{m}$ long pixels with two n-type electrodes and shares six p-type electrodes with its neighbor pixels. The decision for the 2E-250 design with an electrode distance of about $71 \mu \mathrm{m}$ was motivated by studies which were performed with $3 \mathrm{D}$ sensors and different electrode configurations on FE-I3 chips [11] [2]. One important difference between the two design options is that the full 3D applies an active edge whereas the double sided process comes with a guard fence column to control the voltage drop at the sensor edge.

Deep reactive ion etching (DRIE) which is used to create the through-holes for the electrode columns is also used to open through-wafer trenches which surround the whole active area of the sensor. These trenches which are later filled with polysilicon form a doped through-wafer electrode. This electrode surrounding the active area of the sensor builds the active edge and creates a charge sensitive region to the sensor edge [12].

For the double sided 3D design a guard fence structure surrounds the sensor active area. This fence comes in two different flavors for the FBK and CNM detectors. For FBK, it consists of an array of ohmic column electrodes all shorted together in order to make the edge region equipotential, so as to prevent the depletion region spreading from the active area to reach the cut line [13]. Figure 2 shows the CNM design, where a 3D guard ring made of junction column electrodes, in turn surrounded by a frame of ohmic column electrodes enables to sink the edge leakage current [14].

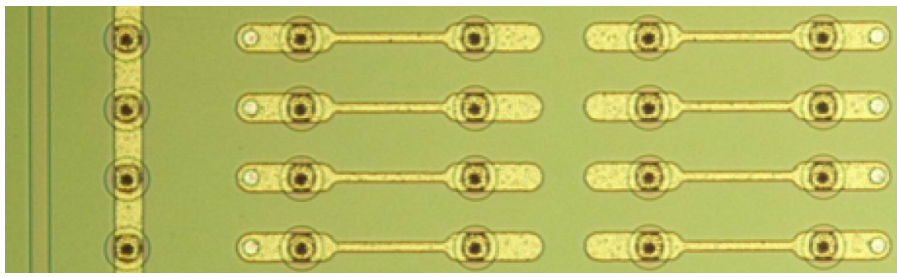

Fig. 2. Pixel layout of a double sided 3D sensor from CNM showing the two electrodes per pixel and the guard ring. The corresponding guard fence structure is placed on the other side of the sensor [15].

In both designs the size of the edge region is $200 \mu \mathrm{m}$ plus an additional $25 \mu \mathrm{m}$ residual of the dicing line. With the FBK edge termination, it has been found that the dead area can be reduced to less than $100 \mu \mathrm{m}$ [16].

Despite the complexity of the 3D production process the collaboration is going to demonstrate that it is possible to produce the sensor with a sufficient high yield of $60 \%$.

The 3D candidate for the upcoming ATLAS IBL is the double sided 3D design with full or partial $(>200 \mu \mathrm{m})$ electrodes produced at Fondazione Bruno Kessler ${ }^{2}$ (FBK) (Trento, Italy) and Centro National de Microelectronice 3 (CNM) (Barcelona, Spain) respectively.

\section{Diamond Pixel Sensors}

Due to the short time scale for the IBL project and the long production time of polycrystalline chemical vapor deposition (pcCVD) diamond material, no diamond Pixel Detector will be used in the Insertable B-Layer. Additional efforts are underway to develop a diamond pixel telescope which applies pixellated pcCVD diamond detectors on FE-I4 front-end chips for measurements at high $\eta$ in the forward direction of the experiment. Nevertheless this technology is a potential candidate for future ATLAS Pixel upgrades.

Diamond sensor material remains a promising detector material with very low leakage current and has the advantage that it needs less cooling than silicon detectors to be operated.

For future detector upgrades CVD diamonds have to overcome a few challenges. One economic related challenge is the high price for the raw material which might reduce in the future due to the growing demand for different physics applications. The diamond sensors smaller signal size compared to silicon could be compensated by special low threshold read-out electronics which gets assisted by the low noise properties in diamond. These properties are related to the smaller dielectric constant which directly affects the capacitance and with that the noise. As long as it is possible to control the front-end chip at sufficient low threshold values a signal to noise ratio of almost 50 for a $5 \cdot 10^{15} \mathrm{n}_{\mathrm{eq}} \mathrm{cm}^{-1}$ irradiated diamond sensor provides enough tolerance to operate diamond detectors at IBL requirements and above [2].

Test beam measurements on samples before and after irradiation have shown that CVD diamond material indeed features the expected radiation hardness. It was demonstrated with FE-I3 modules shown in Figure 3, that diamond sensors can be still operated at SuperLHC (SLHC) fluences of $2 \cdot 10^{16} \mathrm{n}_{\mathrm{eq}} \mathrm{cm}^{-1}$ [17].

The ATLAS Diamond Pixel collaboration is trying to verify that pcCVD diamonds can be produced at the required quality with a reasonable price for future upgrade efforts [2].

\section{IBL IRRADIATION SCHEDULE}

In order to understand the behavior of the different samples after irradiation and especially at the IBL fluence of $5 \cdot 10^{15} \mathrm{n}_{\mathrm{eq}} \mathrm{cm}^{-2}$ several modules have been irradiated at multiple irradiation facilities.

\footnotetext{
${ }^{2}$ Fondazione Bruno Kessler: http://www.fbk.eu

${ }^{3}$ Centro National de Microelectronica: http://www.cnm.es
} 


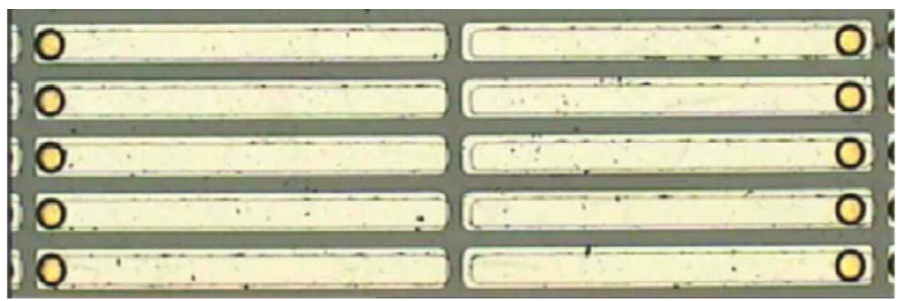

Fig. 3. Pixel pattern with under-bump metalization for a FE-I3 diamond pixel sensor [18]

Three irradiation facilities are available to cover this activities: the Karlsruhe Institute of Technology ${ }^{4}$ (KIT) (Karlsruhe, Germany) provides a low energy proton beam of $25 \mathrm{MeV}$. Due to the low energy beam the ionizing radiation damage exceeds the TID of the FE-I4 by a factor of three for fluences of $5 \cdot 10^{15} \mathrm{n}_{\mathrm{eq}} \mathrm{cm}^{-1}$. This high TID might cause unexpected problems in the front-end chip which need to be understood. First irradiated samples from the Proton Synchrotron (PS) at CERN (Geneva, Switzerland) are expected to be available at the end of 2011. The PS provides as the KIT a proton beam but with an energy of $24 \mathrm{GeV}$.

A neutron irradiation can be done by the n-TRIGA reactor (Ljubljana, Slovenia) at the Jožef Stefan Institute ${ }^{5}$ (JSI) [19].

\section{Measurement Results}

Laboratory measurements have been performed on two planar pixel samples and two double sided 3D pixel samples from CNM. In each case one neutron irradiated and one unirradiated sample were characterized. An overview of the studied assemblies and the different characterization settings are given in Table [I and Table [I] All the presented results have been performed at CERN in an environment chamber at a stable air temperature and low humidity. The quoted temperatures refer to the air temperature in the environment chamber and do not describe the actual sensor temperature.

TABLE I

OVERVIEW OF CHARACTERIZED ASSEMBLIES

\begin{tabular}{cccc}
\hline Device ID & Technology & Fluence & Particle \\
\hline PPS-1 & PPS conservative & not irradiated & \\
PPS-2 & PPS conservative & $3.75 \cdot 10^{15} \mathrm{n}_{\text {eq }} \mathrm{cm}^{-2}$ & Neutron \\
3D-1 & CNM double sided 3D & not irradiated & \\
3D-2 & CNM double sided 3D & $5 \cdot 10^{15} \mathrm{n}_{\mathrm{eq}} \mathrm{cm}^{-2}$ & Neutron \\
\hline
\end{tabular}

The reason for the different fluence between the two irradiated samples is based on the determination of the collected dose which can be only done after the irradiation. Besides the different fluences, there are other differences that make it impossible to directly compare the measured ToT results between the 3D and planar assemblies. On the one hand there were different target tunings applied which affect the ToT

\footnotetext{
${ }^{4}$ Karlsruhe Institute of Technology: http://www.kit.edu

${ }^{5}$ Jožef Stefan Institute: http://www.ijs.si/ijsw/JSI
}

TABLE II

ASSEMBLIES CONFIGURATION INCLUDING TARGET THRESHOLD AND THE TARGET TOT VALUE FOR A GIVEN CHARGE

\begin{tabular}{cccc}
\hline Device ID & Tuning & Thickness & Temperature \\
\hline PPS-1 & $3200 \mathrm{e}^{-}, 5$ ToT at $10 \mathrm{ke}^{-}$ & $250 \mu \mathrm{m}$ & $20^{\circ} \mathrm{C}$ \\
PPS-2 & $1600 \mathrm{e}^{-}, 5$ ToT at $10 \mathrm{ke}^{-}$ & $250 \mu \mathrm{m}$ & $-30^{\circ} \mathrm{C}$ \\
3D-1 & $3200 \mathrm{e}^{-}, 8$ ToT at $20 \mathrm{ke}^{-}$ & $230 \mu \mathrm{m}$ & $20^{\circ} \mathrm{C}$ \\
3D-2 & $1500 \mathrm{e}^{-}, 8$ ToT at $20 \mathrm{ke}^{-}$ & $230 \mu \mathrm{m}$ & $-20^{\circ} \mathrm{C}$ \\
\hline
\end{tabular}

distribution of the assemblies. On the other hand the two irradiated samples experienced different annealing schemes which change the charge collection behavior. Due to an accident in the cooling chain PPS-2 got warmed up to $125^{\circ} \mathrm{C}$ for $10 \mathrm{~min}$ and stayed at room temperature for $46 \mathrm{~h}$. 3D2 was kept at $60^{\circ} \mathrm{C}$ for $120 \mathrm{~min}$ in order to cure the glue between the sample and the PCB. Both samples stayed in a cold environment for the rest of the time in order to prevent further annealing.

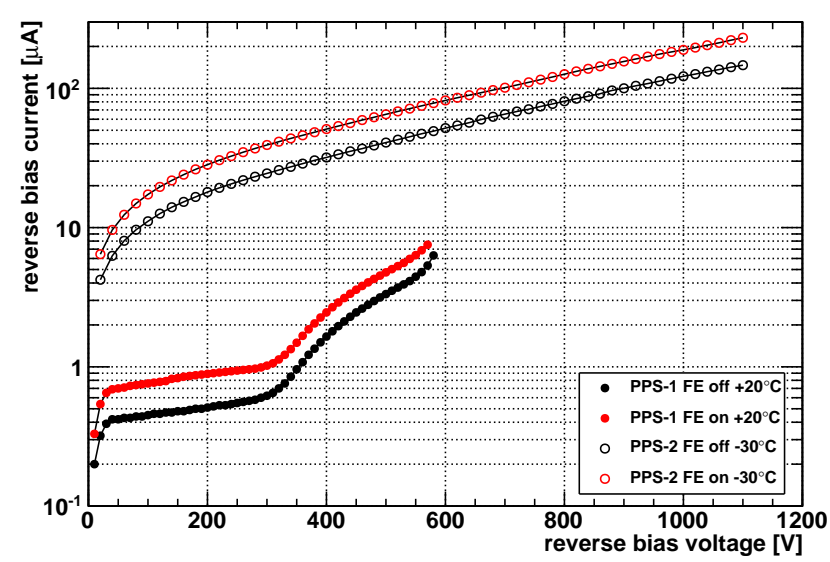

Fig. 4. IV-measurements for unirradiated and irradiated sensors with and without powered front-end chip for (a) PPS and (b) 3D

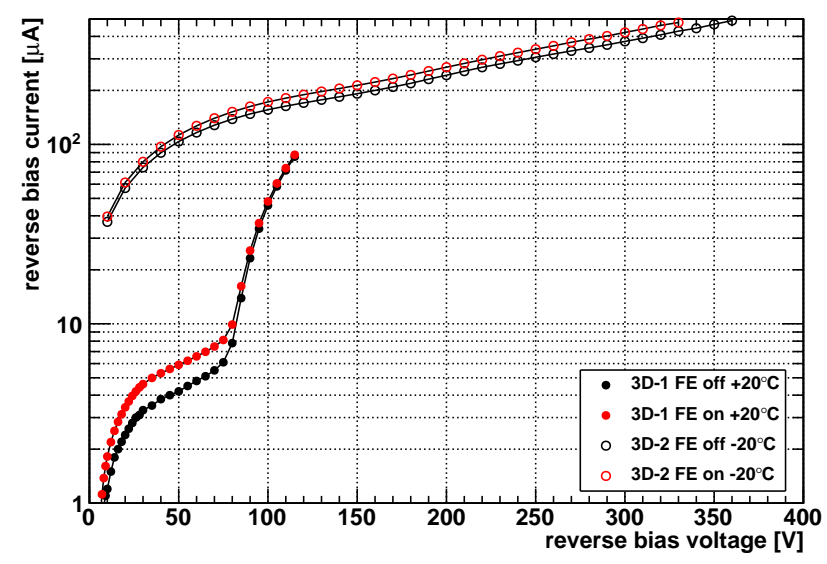

Fig. 5. IV-measurements for unirradiated and irradiated sensors with and without powered front-end chip for (a) PPS and (b) 3D 


\section{A. IV-Measurements}

The measurement of the IV-behavior before irradiation is a common scan to test the device for damages caused during the dicing or the bump bonding process. For irradiated samples it can be also used to monitor the status of the annealing. As the samples needed to be glued and wirebonded after the irradiation it was not possible to perform IV-measurements before the annealing. The induced heat from the front-end chips power dissipation increases the leakage current through the sensor. Therefore, it is necessary to perform the IV-measurements without power at the front-end chip. IV-measurements with and without powered front-end chip can be used to estimate the induced heat in the sensor.

The increased leakage current after irradiation is mostly caused by displacement damage in the silicon sensor. Due to this damage it was necessary to operate PPS-2 and 3D-2 in an environment chamber at $-30{ }^{\circ} \mathrm{C}$ and $-20^{\circ} \mathrm{C}$ respectively in order to keep a stable bias current in the operation range.

The actual applied sensor bias voltage can be calculated by considering the $100 \mathrm{k} \Omega$ resistor connected in series to the sensor and the IV-measurements in Figure 4 and Figure 5 In order to reach the expected operation voltage of around $-160 \mathrm{~V}$ for 3D-2 a bias voltage of $-190 \mathrm{~V}$ needs to be applied to the assembly. Due to the higher bias voltage for PPS- 2 this effect changes the applied voltage only in a range of $2 \%$.

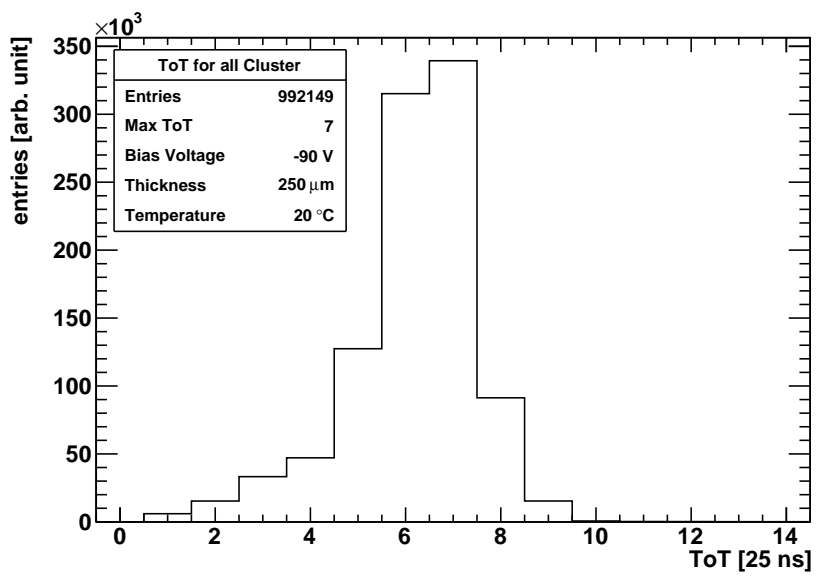

Fig. 6. Photoelectric peak at $60 \mathrm{keV}$ from Am-241 for unirradiated PPS-1

\section{B. Source Measurements}

Measurements with radioactive sources such as Am-241 and $\mathrm{Sr}-90$ are an easy possibility to analyse the sensor performance for devices before and after irradiation.

$\gamma$-sources like Am-241 can be used as a quick way to check the bump-bond connections between the sensor and the front-end chip. As the emitted energies of Am-241 are well known the scan can also be used to confirm the tuning of the device. An external trigger setup with a collimated $\beta$-source as $\mathrm{Sr}-90$ can be used to simulate 'beam like' conditions and to analyze the charge collection of the sensor.

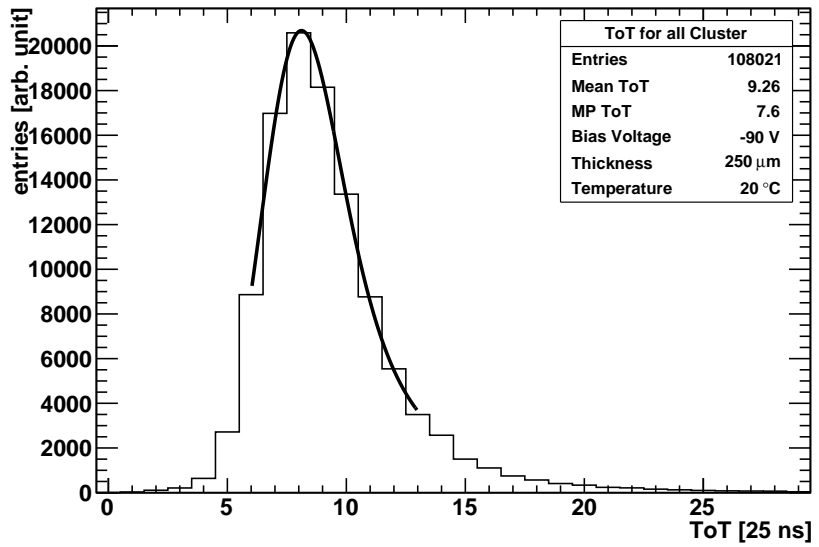

Fig. 7. ToT-spectrum and fit for a convolution of a Landau- and Gaussiandistribution from a Sr-90 source scan with unirradiated PPS-1

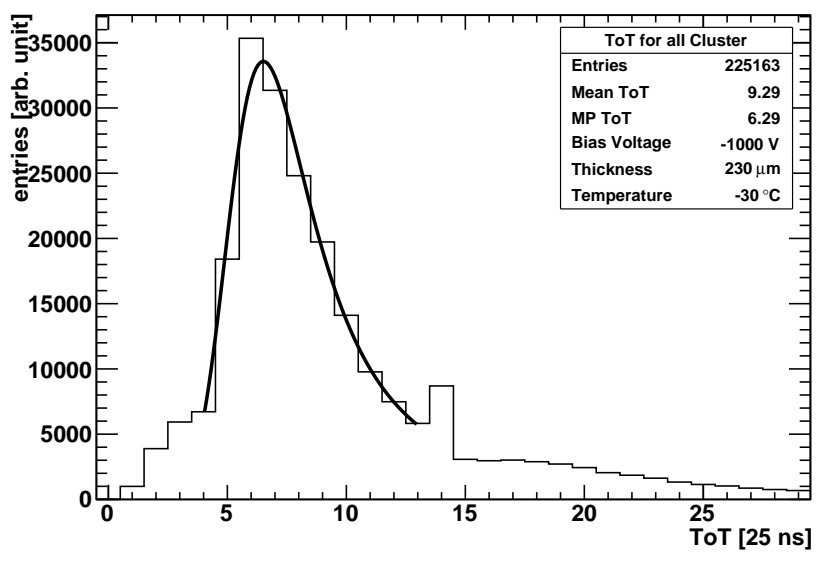

Fig. 8. ToT-spectrum and fit for a convolution of a Landau- and Gaussiandistribution from a Sr-90 source scan with $3.75 \cdot 10^{15} \mathrm{n}_{\mathrm{eq}} \mathrm{cm}^{-2}$ neutron irradiated PPS-2

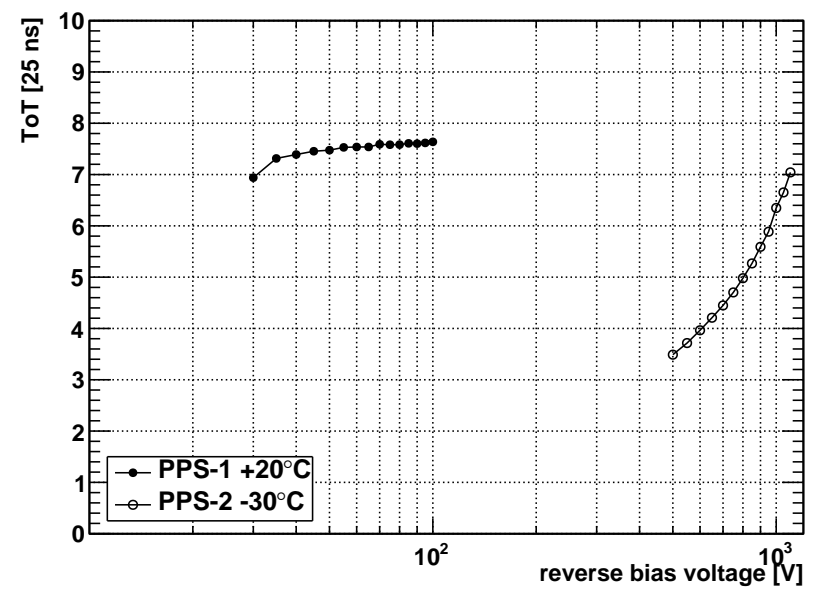

Fig. 9. Most probable ToT value from a Sr-90 source scan in function of the bias voltage for PPS- 1 and PPS-2 
1) Americium - 241: The Am-241 $\gamma$-source emits photons with an energy of $60 \mathrm{keV}$ which deposit a charge of around $16.6 \mathrm{ke}^{-}$in silicon. The measured photoelectric peak can be used to verify the ToT calibration of the device.

As the photoelectron absorbs the whole energy of the penetrating photon the internal hitbus-trigger of the front-end chip has to be used to start the data readout.

The results in Figure 6 and Figure 10 show the measured photoelectric peak for PPS-1 and 3D-1. Both measurements confirm the expected charge collection considering the charge range of $2.0 \mathrm{ke}^{-} \cdot \mathrm{ToT}^{-1}$ to $2.5 \mathrm{ke}^{-} \cdot \mathrm{ToT}^{-1}$ and an estimated error of about $10 \%$ which is related to the injection capacitance. Due to the threshold value of $3200 \mathrm{e}^{-}$and the wide ToT range of the chip it is not possible to see the second Am-241 emission line at $14 \mathrm{keV}$ which would correspond to a charge of $3900 \mathrm{e}^{-}$in silicon.

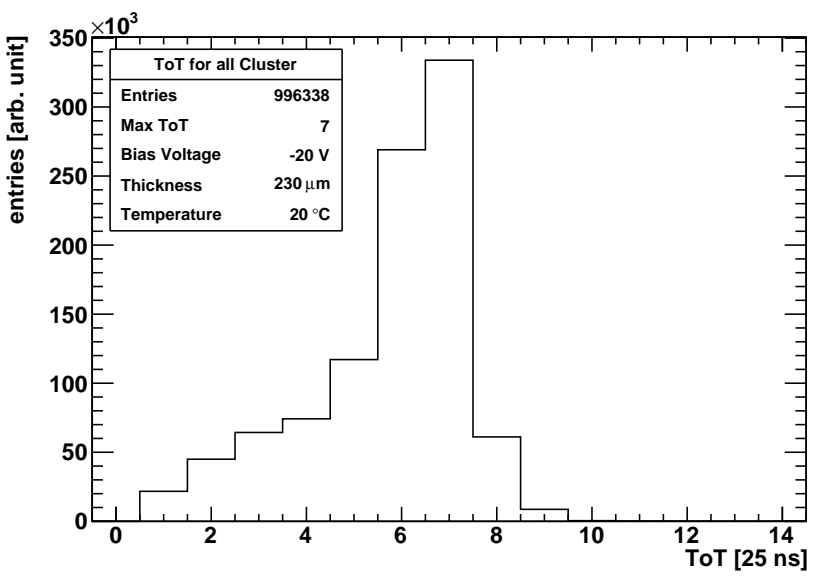

Fig. 10. Photoelectric peak at $60 \mathrm{keV}$ from Am-241 for unirradiated 3D-1

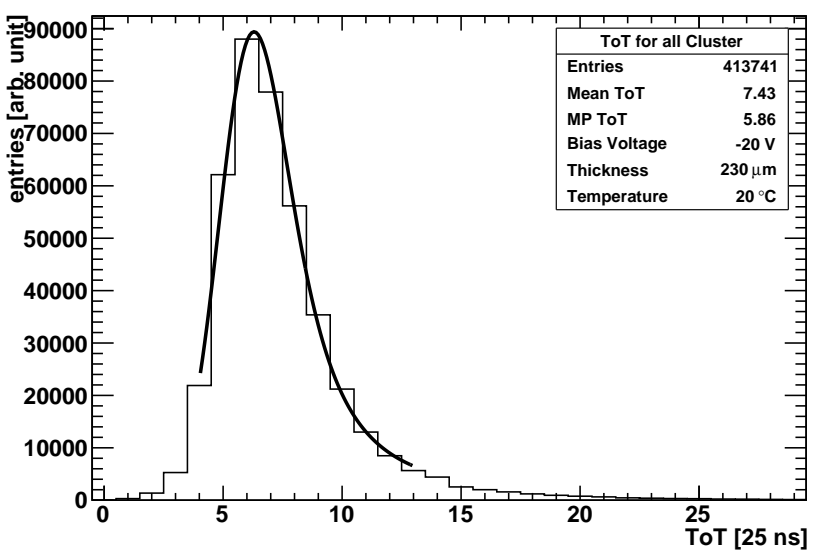

Fig. 11. ToT-spectrum and fit for a convolution of a Landau- and Gaussiandistribution from a Sr-90 source scan with unirradiated 3D-1

2) Strontium - 90: A typical way to analyze the charge collection behavior of a device is to use a $\beta$-source with sufficiently high energy to pass through the whole sample.
$\mathrm{Sr}-90$ is a commonly used radioactive source to simulate a minimum-ionizing particle (MIP) as it decays into Y-90 and later to $\mathrm{Zr}-90$ by emitting $\beta$-particles with an energy of $546 \mathrm{keV}$ and $2.3 \mathrm{MeV}$ respectively. An external trigger setup is used to trigger the data readout only for high energetic electrons with enough energy to penetrate the whole assembly. This setup consists of the Sr-90 source with a brass collimator which is placed on top of a scintillator attached to a photomultiplier. For the measurement the device under test is placed between the source and the scintillator in a way such that the collimated beam passes through the device into the scintillator. Measurements show a beam spot with a width of about $5 \mathrm{~mm}$ which is related to the geometrical conditions of the setup and the $1 \mathrm{~mm}$ thick collimator hole [20].

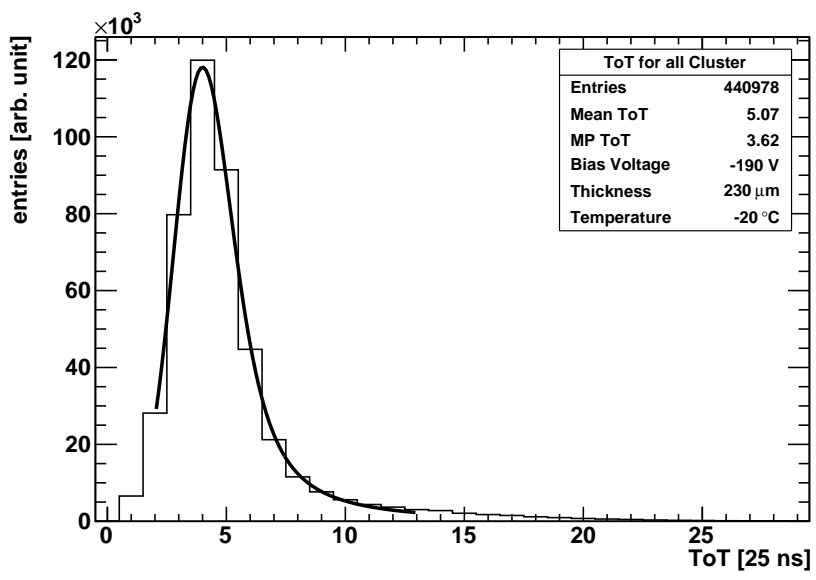

Fig. 12. ToT-spectrum and fit for a convolution of a Landau- and Gaussiandistribution from a Sr-90 source scan with $5 \cdot 10^{15} \mathrm{n}_{\mathrm{eq}} \mathrm{cm}^{-2}$ neutron irradiated $3 \mathrm{D}-2$

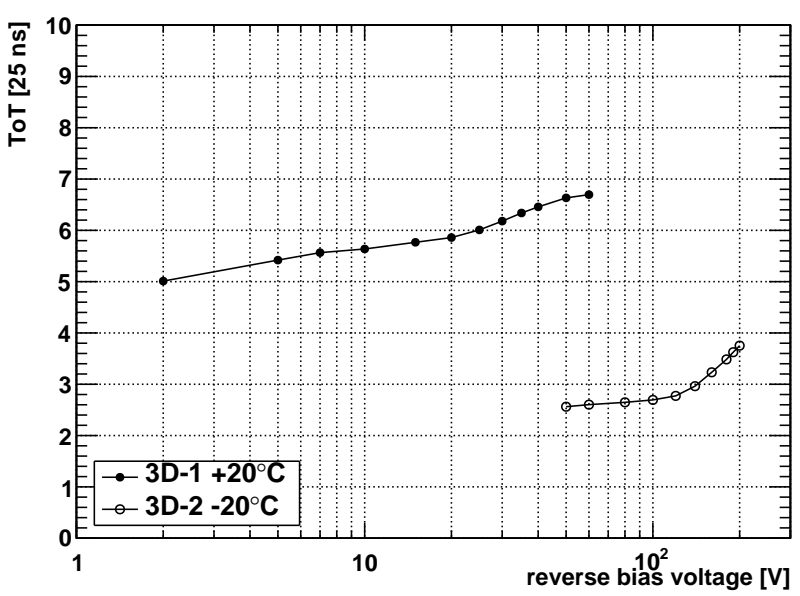

Fig. 13. Most probable ToT value from a $\mathrm{Sr}-90$ source scan in function of the bias voltage for 3D-1 and 3D-2

Figure 7 and Figure 11 show the ToT spectra for both unirradiated devices. The presented distributions represent the ToT spectra for all appeared cluster sizes, with the fits for a 
convolution of a Landau- and Gaussian-distribution. The two measurements confirm the full charge collection for the given operation voltage of $-90 \mathrm{~V}$ for PPS- 1 and $-20 \mathrm{~V}$ for $3 \mathrm{D}-1$. Looking at the cluster size distribution for both samples it occurs that for PPS-1 $35 \%$ and $48 \%$ of all hits are attributed to clusters with a size of one and two pixels whereas the distribution for 3D-1 changes to $50 \%$ for cluster size one and $41 \%$ for cluster size two. This effect is related to the fact that PPS-1 is glued directly on the PCB while 3D-1 is glued on a $500 \mu \mathrm{m}$ thick aluminium plate which covers a FE-I4 shaped hole in the PCB. As the emitted electrons from the $\mathrm{Sr}$ 90 source have to pass through the roughly $1 \mathrm{~mm}$ thick PCB of PPS-1 low energetic electrons get filtered out. Due to the smaller thickness of the aluminium plate this effect is less in 3D-1.

The ToT spectra for the two neutron irradiated assemblies PPS-2 in Figure 8 and 3D-2 in Figure 12 show the reduced charge collection after irradiation. The cluster size distributions for PPS-2 and 3D-2 show that roughly $16 \%$ more hits with a cluster size one appear in 3D-2. This effect is related to the different fluences of the two devices.

As an example a particle passing trough two pixels deposits charge related to the length of the way in both pixels. If the deposited charge in one of the pixels is below the threshold value this cluster with a real cluster size of two pixels gets considered as a one pixel cluster with a lower charge. This effect increases for higher fluences as the deposited charge in the second pixel decreases due to the irradiation damage. The difference in the cluster size distribution between the two 3D samples can be also traced back to the influence of this defect. It is difficult to compare the obtained results in the case of the PPS samples as two effects are overlapping since PPS- 1 is not glued on an aluminium plate.

The excess of entries in TOT bin 14 in Figure 8 can be considered as an artifact of the 4-bit ToT resolution in the front-end chip. This artifact is related to the clustering and the maximal ToT value of 14 .

The results for the most probable ToT value depending on the applied bias voltage for unirradiated and irradiated devices are shown in Figure 9 and Figure 13. The saturation region starting at about $-40 \mathrm{~V}$ for PPS- 1 and $-10 \mathrm{~V}$ for 3D-1 indicates that both sensors are fully depleted. For lower voltages the sensors stay in an under-depleted state where it is not possible to collect the full charge.

Looking at PPS-2 the obtained most probable ToT values agree well with already published results for a fluence of $3.75 \cdot 10^{15} \mathrm{n}_{\mathrm{eq}} \mathrm{cm}^{-2}$ and an operation voltage of $-900 \mathrm{~V}$. A signal efficiency of $83 \%$ can be achieved by comparing the collected ToT results of PPS-1 and PPS-2 [2]. Comparing 3D-1 before irradiation and 3D-2 after irradiation to $5 \cdot 10^{15} \mathrm{n}_{\mathrm{eq}} \mathrm{cm}^{-2}$ it can be observed that the signal efficiency after irradiation is still at about $62 \%$ which confirms results obtained with similar 3D sensors [2].

\section{CONCLUSION}

First characterization results on pixel sensors bump-bonded to FE-I4 chips have been discussed. Measurements to analyze the IV-behavior and the charge collection properties of the samples have been studied and comparisons of the behavior before and after irradiation have been performed. Both sensor designs behave as expected considering the analyzed topics. Pion and electron test-beam measurements from CERN and DESY for both sensor technologies will soon be available in order to understand the sensor behavior for real MIPs.

Additional samples from both technologies will be irradiated to fluences higher and lower than the expected fluence in IBL. Further statistics will be gathered to understand the radiation hardness for levels at the following ATLAS Pixel Detector upgrade.

\section{ACKNOWLEDGMENT}

We would like to thank A. Dierlamm (KIT) and M. Glaser (CERN) for carrying out the device irradiation in Karlsruhe (Germany) and CERN PS (Switzerland) respectively. We would also like to thank V. Tromel and Y. Donjoux from the CERN Radiation Protection Group for the support and assistance concerning the manipulation of irradiated material. Additional thanks to I. McGill for the wire-bonding of the irradiated samples.

\section{REFERENCES}

[1] F. Gianotti et al., "Physics potential and experimental challenges of the LHC luminosity upgrade," Eur. Phys. J. C, vol. 39, pp. 293-333, 2005.

[2] The ATLAS Collaboration, "ATLAS Insertable B-Layer Technical Design Report," CERN, Geneva, Tech. Rep. CERN-LHCC-2010-013. ATLAS-TDR-019, Sep 2010.

[3] G. Aad et al., "ATLAS pixel detector electronics and sensors," JINST, vol. 3, p. P07007, 2008.

[4] M. Garcia-Sciveres et al., "The FE-I4 pixel readout integrated circuit," Nucl. Instrum. Methods A, vol. 636, pp. 1-5, Apr 2010.

[5] D. Muenstermann, C. Goessling, R. Klingenberg, and T. Wittig, "Evaluation of the breakdown behaviour of ATLAS silicon pixel sensors after partial guard-ring removal," Nucl. Instrum. Methods A, vol. 624, no. 2, pp. 410-413, Dec 2010.

[6] M. Benoit, A. Lounis, and N. Dinu, "Simulation of guard ring influence on the performance of ATLAS pixel detectors for inner layer replacement," JINST, vol. 4, no. 03, pp. P03 025-P03 025, Mar 2009.

[7] — " "Simulation of Radiation Damage Effects on Planar Pixel Guard Ring Structure for ATLAS Inner Detector Upgrade," IEEE Trans. Nucl. Sci., vol. 56, no. 6, pp. 3236-3243, Dec 2009.

[8] T. Wittig et al., "Planar slim-edge pixel sensors for the ATLAS upgrades," Sep 2011, in preparation for PSD9: Position Sensitive Detectors Conference - Aberystwyth (UK).

[9] P. Weigell et al., "Characterization and performance of silicon n-in-p pixel detectors for the atlas upgrades," Nucl. Instrum. Methods A, vol. In Press, Corrected Proof, 2011.

[10] S. Parker, C. J. Kenney, , and J. Segal, "3D: A proposed new architecture for solid-state radiation detectors," Nucl. Instrum. Methods A, vol. 395, no. 3, pp. 328-343, Aug 1997.

[11] C. Da Via et al., "3D active edge silicon sensors with different electrode configurations: Radiation hardness and noise performance," Nucl. Instrum. Methods A, vol. 604, pp. 505-511, 2009.

[12] T. Hansen et al., "First fabrication of full 3D-detectors at SINTEF," JINST, vol. 4, no. 3, pp. P03 010-P03 010, Mar 2009.

[13] G.-F. Della Betta et al. , "Development of modified 3D detectors at FBK," in Nuclear Science Symposium Conference Record (NSS/MIC), 2010 IEEE, Nov 2010, pp. 382-387.

[14] C. Fleta et al., "Laboratory and Testbeam Results on 3D Detectors," in Proceedings of the 19th International Workshop on Vertex Detectors. June 6-11, 2010, Loch Lomond, Scotland, UK, vol. 1, 2010, p. 23.

[15] G. Pellegrini et al., "First double-sided 3-D detectors fabricated at CNMIMB," Nucl. Instrum. Methods A, vol. 592, no. 1-2, pp. 38 - 43, 2008.

[16] M. Povoli et al., "Slim edges in double-sided silicon 3D detectors," Jul 2011, presented at IWORID 2011, Zurich (CH). 
[17] M. Cristinziani, "Diamond prototypes for the ATLAS SLHC pixel detector," Nucl. Instrum. Methods A, vol. 623, no. 1, pp. 174-176, Nov 2010.

[18] W. Adam et al., "Radiation hard diamond sensors for future tracking applications," Nucl. Instrum. Methods A, vol. 565, no. 1, pp. 278-283, Sep 2006.

[19] A. La Rosa, "ATLAS IBL: a challenging first step for ATLAS Upgrade at the sLHC," CERN, Geneva, Tech. Rep. ATL-INDET-SLIDE-2011346, Jul 2011.

[20] S. Altenheiner et al., "Radiation hardness studies of $n+-i n-n$ planar pixel sensors for the ATLAS upgrades," Nucl. Instrum. Methods A, pp. 1-5, Jun 2011. 